\title{
0D/2D Heterostructures Vertical Single Electron Transistor
}

Louis Donald Notemgnou Mouafo*, Florian Godel, Laurent Simon*, Yannick J. Dappe*, Walid Baaziz, Ulrich Nguétchuissi Noumbé, Etienne Lorchat, Marie-Blandine Martin, Stéphane, Berciaud, Bernard Doudin, Ovidiu Ersen, Bruno Dlubak, Pierre Seneor*, Jean-Francois Dayen*

Dr. L.D.N. Mouafo ${ }^{1}$, Dr. W. Baziz ${ }^{1}$, U.N. Noumbé ${ }^{1}$, Dr. E. Lorchat ${ }^{1}$, Pr. S. Berciaud ${ }^{1,2}$, Pr. B. Doudin $^{1}$, Pr. O. Ersen ${ }^{1}$ and Dr. Jean-Francois Dayen ${ }^{1,2}$

${ }^{1}$ Université de Strasbourg, CNRS, Institut de Physique et Chimie des Matériaux de Strasbourg (IPCMS), UMR 7504, 23 rue du Loess, Strasbourg, 67034, France.

${ }^{2}$ Institut Universitaire de France, 1 rue Descartes, 75231 Paris cedex 05.

E-mail: donald.1.mouafo@gmail.com,dayen@unistra.fr

Dr. F. Godel ${ }^{3}$, Dr. M.-B. Martin ${ }^{3}$, Dr. B. Dlubak ${ }^{3}$ and Pr. P. Seneor ${ }^{3}$

${ }^{3}$ Unité Mixte de Physique, CNRS, Thales, Université Paris-Saclay, 91767 Palaiseau, France

E-Mail: pierre.seneor@cnrs-thales.fr

Dr. L. Simon ${ }^{4}$

${ }^{4}$ Institut de Sciences des Matériaux de Mulhouse, CNRS-UMR 7361, Université de Haute Alsace, 68093 Mulhouse, France.

E-Mail: laurent.simon@uha.fr

Dr. Y. J. Dappe ${ }^{5}$

${ }^{5}$ SPEC, CEA, CNRS, Université Paris-Saclay, CEA Saclay, 91191 Gif-sur-Yvette Cedex

France.

E-Mail: yannick.dappe@cea.fr

Keywords: two dimensional materials, transition metal dichalcogenides, nanoparticule, single electron transistor, heterostructure, nanoelectronics, multifunctional materials.

Abstract : Mixed-dimensional heterostructures formed by the stacking of 2D materials with nanostructures of distinct dimensionality, constitute a new class of nanomaterials that offers multi-functionality that goes beyond those of single dimensional systems. An unexplored architecture of Single Electron Transistor (SET) is developed, that employs heterostructures made of nanoclusters (0D) grown on a two dimensional (2D) Molybdenum disulfide $\left(\mathrm{MoS}_{2}\right)$ channel. Combining the large Coulomb energy of the nanoclusters with the electronic 
capabilities of the 2D layer, the concept of 0D-2D Vertical SET is unveiled. The $\mathrm{MoS}_{2}$ underneath serves both as a charge tunable channel interconnecting the electrode, and as bottom electrode for each v-SET cell. In addition, its atomic thickness makes it thinner than the Debye screening length, providing electric field transparency functionality that allows for an efficient electric back gate control of the nanoclusters charge state. Coulomb diamond patterns characteristics of SET are reported, with specific doping dependent non-linear features arising from the $0 \mathrm{D} / 2 \mathrm{D}$ geometry that are elucidated by theoretical modeling. These results hold promise for multifunctional single electron device taking advantage of the versatility of the $2 \mathrm{D}$ materials library, with as example envisioned spintronics applications while coupling quantum dots to magnetic 2D material, or to ferroelectric layers for neuromorphic devices.

\section{Introduction}

Two-dimensional (2D) materials have triggered intensive research activities over the past few years with yet foreseeable prospect for both fundamental and applicative studies. ${ }^{[1,2]}$ Reduced to a single or few layers, their atomically thin structure and low density of states (DOS) favor their transparency to electric field. In general, the electric field penetrates a material over a distance of the order of the Debye screening length, which gets shorter for higher carrier density. In the case of a bulk metal, the important carrier density $\left(\sim 10^{22} \mathrm{~cm}^{-3}\right)$ limits the Debye screening length below the Angstrom such that the electric field is completely screened at the surface. The 2D nature of lamellar materials results in specific band structure and quantum capacitance properties ${ }^{[3,4]}$ that prevent charge to reorganize along the perpendicular axis to the surface to screen vertical static electrical field. This intrinsic limitation combined with their rather low carrier density $\left(\sim 10^{12} \mathrm{~cm}^{-2}\right)$ results in a Debye screening length reaching up to several nanometers, ${ }^{[5,6]}$ making therefore $2 \mathrm{D}$ materials transparent to the electric field, even for thickness of several monolayers. New properties and functionalities are expected to emerge 
when coupling $2 \mathrm{D}$ materials with nanostructures of distinct low dimensionality $\mathrm{n}_{\mathrm{D}}$ (equals to $0,1$, or 3$)$, driven by the electric modulation of either the $\mathrm{nD} / 2 \mathrm{D}$ interfaces and/or the electronic properties of the individual constituent of such heterostructures referred as mixed-dimensional van der Waals heterostructures (m-vdWh). ${ }^{[7,8]}$

In addition to opening doors to novel device geometries, m-vdWh offer attractive properties such as highly tunable electronic band profile at the $\mathrm{nD} / 2 \mathrm{D}$ system useful for field effect transistors and photodetectors ${ }^{[8-11]}$, hybridized interfaces with unique spintronic properties ${ }^{[12-}$ ${ }^{18]}$ and proximity induced quantum properties. ${ }^{[19-22]}$ Though many of these devices are fabricated by depositing nanomaterials over the surface of the 2D material using either vacuum deposition, ${ }^{[23-25]}$ drop casting or coating, ${ }^{[7,11,26]} 2 \mathrm{D}$ materials have also demonstrated to be promising template for the growth of self-assembled nanoclusters (NC) providing alternative route toward new fabrication methods with proven promises in catalysis, ${ }^{[2]}$ magnetic memory, ${ }^{[14,28]}$ quantum devices ${ }^{[19,22]}$ and device engineering. ${ }^{[29]}$

One fascinating property of nanostructure and especially nanoparticles, is their large Coulomb charging energy which enables to develop nanodevice that demonstrate control of the charge transfer process at the single electron level. Hence, combining the single electron transport functionality of nanoparticles with the unique electronic properties of $2 \mathrm{D}$ materials is a seducing approach to explore alternative architectures of single electron devices. 0D/2D m-vdWh provide several advantages compared to conventional materials such as $\mathrm{Si}, \mathrm{Ge}, \mathrm{GaAs}$ and related semiconductors based epitaxial heterostructures. First, because of the transparency of the 2D materials to vertical applied electric fields, novel concepts of vertical device architecture can be designed that are not possible with semiconducting and metallic thin films. Second, because of the van der Waals nature of interfaces, a large panel of different materials can be stacked, without the lattice mismatch or physico-chemical restrictions usually encountered with epitaxial or thin film growth technologies. Finally, while enabling the use of bottom-up 
strategies for the design of the Coulomb island element, it circumvents some of the limitations of the top down approach in the design of nanometer-sized objects, especially when 0D metallic nanostructures are pursued.

However, no report has yet highlighted $\mathrm{m}$-vdWh based quantum devices demonstrating gate modulated single electron logic. Yet, recently, Single Electron Transistor (SET) has been demonstrated on 2D/2D system made of $\mathrm{hBN} /$ graphene/hBN stacking, and relied on the exploitation of resonant tunnelling of charges through discrete energy states of impurities present in the h-BN barrier. ${ }^{[30]}$ Likewise, the encapsulation of single layer graphene (Gr) into insulating h-BN used as dielectric allowed engineering electrically depleted quantum dot with remarkable Coulomb blockade (CB) transport properties. ${ }^{[31-33]}$ However, this requires state of the art e-beam lithography combined with mechanical Van der Waals staking techniques known to be challenging and not yet scalable. Besides, despite the low relative permittivity of h-BN, the charging energy remains usually limited to few Kelvin range. ${ }^{[31,32]}$

In previous contributions, we reported that core(metal)/shell(insulator) nanoparticle of $\mathrm{Al}($ core $) / \mathrm{AlOx}($ shell) can be self-orderly grown over a graphene template by electron beam evaporation of a very thin Al layer $(<2 \mathrm{~nm})$ followed by oxidation in air. ${ }^{[14,22]}$ These unique 0D/2D heterostructures are a suitable architecture to develop two terminals single electron device demonstrating robust conductance oscillation at low temperature. In addition, this system has shown remarquable single electron spintronics functionalities making use of spinorbit assisted magneto-Coulomb effect. ${ }^{[14]}$ However, to date, the implementation of such 0D/2D heterostructures into three terminals single electron transistor, the building block of single electron logic circuit, remains to be shown.

In this report, we demonstrate a simple fabrication route and the operation of vertical SET (vSET) using 0D/2D m-vdWh consisting of oxidized self-assembled Al nanoclusters grown over 
$\mathrm{MoS}_{2}$ template (Figure 1) exfoliated on $\mathrm{Si} / \mathrm{SiO}_{2}$ back gate substrate. Here we take advantage of the electric field controlled doping of the 2D semiconductor templates, to unveil the tunable single electron transistor functionalities of a new family of $0 \mathrm{D} / 2 \mathrm{D}$ heterostructures. The $\mathrm{MoS}_{2}$ underneath serves both as a doping tunable channel interconnecting the electrode, and as bottom electrode for each v-SET cell (Figure 2). In addition, its atomically thin structure and semiconductor character give it better electric field transparency, providing an efficient electric back gate control of the nanoclusters charge state organized over its surface (Figure 3). In the following, we report experimental and theoretical modeling results (Figure 4), that reveal how this original device architecture enables achieving low temperature single electron transport functionalities with three terminals charge stability diagrams demonstrating Coulomb diamond patterns characteristics of SET with specific doping dependent non-linear characteristics arising from the 0D/2D geometry. These results demonstrate how the 0D/2D v-SET architecture can capture in a single device the functionalities of a field effect transistor with high on/off ratio and rectifying characteristics, with the Coulomb blockade charge properties of a SET with tunable electrical parameters. Lastly, the CB features in v-SET are still observed up to relatively high temperature (50K). Realistic improvements of our sample design could allow the demonstration of v-SET operating at room temperature, encouraging effort to make for the next generation of functional ambient temperature v-SET (Figure 5).

\section{Results and discussion}

\subsection{D-2D heterostructures growth and material characterization}

The $\mathrm{MoS}_{2}$ flakes are prepared on a clean $\left(\mathrm{p}^{++}\right) \mathrm{Si}_{/} / \mathrm{SiO}_{2}(285 \mathrm{~nm})$ surface, using conventional mechanical exfoliation method (see Method). ${ }^{[34]}$ We present the Raman spectrum recorded 
using a laser with photon energy at $2.33 \mathrm{eV}$ (wavelength of $532 \mathrm{~nm}$ ). The red and black dots on the inserted optical image of the flake indicate the location of the Raman spots corresponding to the red and black curves respectively (Figure 1a). On the red curve, two ultralow frequency features near $23 \mathrm{~cm}^{-1}$ and $40 \mathrm{~cm}^{-1}$ are assigned to the rigid-layer shear mode ('LSM') and breathing mode ('LBM') of a $\mathrm{MoS}_{2}$ bilayer (with $E_{g}$ and $A_{1 g}$ symmetry), respectively. ${ }^{[35,36]}$ In contrast, no observable rigid-layer modes appear on the black curve, as expected for a $\mathrm{MoS}_{2}$ monolayer. At higher frequency, we observe two well-documented one-phonon intralayer modes. One in-plane mode with $E_{g}$ (resp. $E^{\prime}$ ) symmetry appears at $385 \mathrm{~cm}^{-1}$ (resp. $\left.386 \mathrm{~cm}-1\right)$ in bilayer and monolayer $\mathrm{MoS}_{2}$, respectively. One out-of-plane mode with $A_{1 g}$ (resp. $A_{1}^{\prime}$ ) symmetry appears at $407 \mathrm{~cm}^{-1}$ (resp. $405 \mathrm{~cm}^{-1}$ ) in bilayer and monolayer $\mathrm{MoS}_{2}$, respectively. The significantly smaller frequency difference between these two modes observed in the monolayer flake $\left(\approx 19 \mathrm{~cm}^{-1}\right.$ as compared to $\approx 22 \mathrm{~cm}^{-1}$ in the bilayer flake) further confirm our layer-number assignment. ${ }^{[37]}$

To investigate the structural organization and composition of aluminium oxide barrier on $\mathrm{MoS}_{2}$ platform, we deposited a thin Al layer of $1.7 \mathrm{~nm}$ nominal thickness on top of $\mathrm{MoS}_{2}$. The ultrathin oxidized Al layer is characterized by Scanning Transmission Electron Microscopy (STEM) and presents a granular structure with Al NCs self-assembled on the surface of the $\mathrm{MoS}_{2}$ layer (Figure 1b). The statistical analysis of the NCs lateral diameter (inset of Figure 1b) demonstrates a distribution well approximated by a Gaussian function with a peak at 7.4 $\mathrm{nm}$ and the full-width-at-half-maximum close to $=3.0 \mathrm{~nm}$ which corresponds to $20 \%$ size distribution, consistently with $0 \mathrm{D} / 2 \mathrm{D}$ growth characteristics observed previously on graphene template. ${ }^{[14,22]}$ Such morphology is reminiscent of a 3D growth process. During the $\mathrm{Al}$ evaporation, once adsorbed onto the $\mathrm{MoS}_{2}$ surface, $\mathrm{Al}$ atoms diffuse to the favourable 
nucleation sites probably conditioned both by surface tension and the atomic diffusion rate. This growth process is also consistent with other experiment demonstrating that for a nominal thickness below $2 \mathrm{~nm}$, the growth of $\mathrm{Al}$ on graphene follows a Stranski-Krastanov 2D growth before $3 \mathrm{D}$ clustering leading to uniformly distributed self-assembled nanoclusters. ${ }^{[38]}$ The impact of the aluminium oxidation on the size and shape of clusters, in the earlier stages of oxidation just after Al deposition even under High vacuum conditions, remains an open question.

In order to understand the nature of the oxidation process and the interface between $\mathrm{Al}$ based nanoclusters and the $\mathrm{MoS}_{2}$ substrate, we have performed careful systematic analysis by X-ray photoelectron spectroscopy (XPS), Angle-resolved photoemission spectroscopy (ARPES), and Density Functional Theory (DFT) calculations (see complete combined analysis in Supporting Information). We have followed the nominal parameters used for the fabrication of the device (typically oxidation in air for an $\mathrm{Al}$ deposition thickness of 0.5 to $2 \mathrm{~nm}$ ), with a control of the surface at each step of the process: the preparation of pristine $\mathrm{MoS}_{2}$, the deposition of $\mathrm{Al}$ and the oxidation are done with the transfer of the sample in different Ultra High Vacuum (UHV) chambers without breaking the vacuum. In our system, oxygen $\left(\mathrm{O}_{2}\right)$ exposure can be done from few Langmuir to 0.5 bar of pressure. The pressure is measured using a cold-cathode gauge which allows to follow the pressure from $5.10^{-9}$ mbar to 100 mbar. We provide in Methods and Supporting Information details on the sample and surface preparation steps done under UHV environment prior deposition of $\mathrm{Al}$ and oxidation. Figure 1c and Figure 1d show respectively XPS measurement of the core level peak $\mathrm{Al}_{2 \mathrm{p}}$ just after the deposition of $0.5 \mathrm{~nm}$ of $\mathrm{Al}$ at room temperature and after 60 min exposure to 70 mbar oxygen pressure. We identify A12p metallic doublet with A12p (3/2) (72.82 eV) and A12p (1/2) (73.24 eV) with a spin-orbit splitting of 
$0.42 \mathrm{eV}$ and an intensity ratio of 0.52 . Just after deposition and even under UHV conditions, due to the high reactivity of $\mathrm{Al}$, we always have residual oxide component $\mathrm{AlO} 3+$ observed around $75.8 \mathrm{eV}$. Between this $\mathrm{AlO} 3+$ component and Al metallic we clearly resolve a supplementary component around a binding energy of $73.6 \mathrm{eV}$ which is attributed to an interfacial state (AlOxAl-S) between $\mathrm{MoS}_{2}$ and aluminium oxide. Then, our detailed study of this interface suggests S-Al bonds at the interface between the cluster and the $\mathrm{MoS}_{2}$ surface. Even after oxidation in air, Al metal component together with interfacial state are still visible (see Supporting Information, Figure S1c). In order to fully characterize this $\mathrm{Al}-\mathrm{MoS}_{2}$ interface, we have performed DFT calculations. To this end, we have used the very efficient localized orbital basis set DFT code Fireball. ${ }^{[39]}$ Optimized basis sets for Mo, S, Al and $\mathrm{O}$ have been used, in agreement with previous calculations. ${ }^{[40,41]}$ Hence, we have considered a $5 \times 5$ layer of $\mathrm{MoS}_{2}$, with an Al-O dimer on top, connected to a sulfur atom through either the aluminium atom or the Oxygen one. Both structures have been optimized, and as a result, the $\mathrm{S}-\mathrm{Al}$ interface appears to be more stable energetically than the $\mathrm{S}-\mathrm{O}$ interface for about $2 \mathrm{eV}$. Good agreement is also observed between the band structure calculation of the $\mathrm{MoS}_{2}(\mathrm{~S}-\mathrm{Al}-\mathrm{O})$ system and ARPES measurements (see details in Supporting Information, Figure S3).

We conclude from these combined STEM, XPS and DFT studies that the oxidation process of our ultrathin aluminium layer leads to the formation of core-shell nanostructures with a metallic core and an $\mathrm{Al}_{2} \mathrm{O}_{3}$ shell. In addition, the interface barrier between aluminium and $\mathrm{MoS}_{2}$ originates from the Al-S bonding, the oxidization process taking place at the surface of the deposited aluminium.

\subsection{D-2D single electron transistor transport properties}


Electrode stripes are patterned onto monolayer $\mathrm{MoS}_{2}$ flake presented in Figure 2a by ebeam lithography into a standard bilayer resist (see Methods). We deposited, in same conditions as presented before using e-beam technique, a thin Al layer of $1.7 \mathrm{~nm}$ nominal thickness to form a granular layer of self-assembled $\mathrm{Al}$ nanoclusters (NC) over the $\mathrm{MoS}_{2}$ surface through the electrode pattern. The $\mathrm{MoS}_{2}$ channel in between the electrode remains protected by the polymer resist and is not exposed to the aluminium. The sample is then taken out of the vacuum chamber for oxidation in ambient atmosphere (see reference ${ }^{[14]}$ for details). E-beam evaporation of 40 $\mathrm{nm}$ thick Cobalt capped by $10 \mathrm{~nm}$ of Au completes the electrode stack. Side electrodes made of $\mathrm{Ti}(3 \mathrm{~nm}) \mid \mathrm{Au}(47 \mathrm{~nm})$ electrodes in direct contact with the $\mathrm{MoS}_{2}$ channel are also patterned. Figure 2a shows an optical image of the device after lift-off in acetone. As represented in the scheme of Figure 2b, the $\mathrm{MoS}_{2}$ flake is used both as a semiconducting channel interconnecting the various electrodes (gold electrodes and v-SET cells), and as a bottom electrode part of the 0D/2D v-SET cell(s). Each v-SET cell consists of a stack of Au/Co top electrode contacting the underneath $\mathrm{MoS}_{2}$ through the granular oxidized Al layer.

In the following, we study the single electron properties of such $0 \mathrm{D} / 2 \mathrm{D}$ heterostructures implemented in vertical single electron transistor (v-SET) device geometry. Figure 2 b shows the schematic representation of the 0D/2D SET device. A typical three terminals 0D/2D v-SET can then either consist of one v-SET cell linked via the $\mathrm{MoS}_{2}$ to one Ti/Au electrode forming a Schottky contact, ${ }^{[42]}$ or two v-SET cells interconnected through the $\mathrm{MoS}_{2}$ channel. We refer to these distinct configurations as "Single" and "Twin" cells respectively. The capacitive coupled $\left(\mathrm{p}^{++}\right)$-Si back gate electrode enables a concomitant modulation of both the energy band structure of the underneath $\mathrm{MoS}_{2}$ and the electric state of the supported Al NCs, while exploiting the transparency of the 2D material to the applied vertical electric field. In the v-SET cell, charges are injected from the top metallic electrode to the bottom $\mathrm{MoS}_{2}$ electrode through the oxidized Al nanoclusters. We now investigate the transport properties of our devices. 
Figure 3 presents the low temperature transport properties of devices in Single (Figure 3a,b) and Twin (Figure 3c,d) cells. The Isd-Vsd characteristics (black curves, left axis) and the corresponding differential conductance traces ( $\mathrm{dI} / \mathrm{dVsd}-\mathrm{Vsd}$, red curves and right axis) of both device configurations present a pronounced non-linear trend typical to semiconductor Transition Metal Dichalcogenide (TMD) Field Effect Transitor (FET) and a noticeable asymmetry stemming from differences in source and drain electrodes. ${ }^{[22,43]}$ In the case of the Single cell, such differences relate to the distinct materials used for each contact. For the Twin configuration, the different contact areas and unavoidable slight variations of the tunnel junctions result in the observed asymmetric behaviour. ${ }^{[42,44,45]}$ Notice that the $\mathrm{MoS}_{2}$ channel length has a direct influence on the device characteristics, with much larger device resistance observed for longer $\mathrm{MoS}_{2}$ channel length $(3.5 \mu \mathrm{m}$ in asymmetric device of Figure $3 \mathrm{c})$ compared to configuration with $350 \mathrm{~nm}$ long channel (device of Figure 3a).

Superimposed to the non-linear trend, the Isd-Vsd curves show clear Coulomb staircases features providing evidence of single electron transport mechanisms. These single electron features are better highlighted in the $\mathrm{dI} / \mathrm{dV}$ sd-Vsd curves as well marked peaks for both dc bias polarities (red curves, Figure 3a,c). The Figure 3b,d present the devices current transfer characteristics Isd-Vg while tuning the back gate voltage Vg. A negligible modulation of the current is observed at low gate voltage, indicating that the Fermi energy $\mathrm{E}_{\mathrm{F}}$ of the $\mathrm{MoS}_{2}$ is situated deep within the bandgap. Thus, a minimum threshold gate voltage of $\mathrm{V}_{\mathrm{TH}}=19 \mathrm{~V}$ is required to drag $\mathrm{E}_{\mathrm{F}}$ closer to the vicinity of minimum conduction band edges and trigger the conduction through the $\mathrm{MoS}_{2}$ channel. Beyond $\mathrm{V}_{\mathrm{TH}}$, the current exponentially increases driven by the electrostatic doping of the channel. Interestingly, as the channel becomes conducting we find, superposed to the typical n-type field effect transconductance, remarkable aperiodic current oscillations controlled by the gate voltage (Figure 3b,d). It thus appears that the 
transport of our devices is governed by the superposition of two effects : the field effect modulation of the semiconducting $\mathrm{MoS}_{2}$ channel resistance, and the Coulomb blockade mediated single electron tunnelling at the $0 \mathrm{D} / 2 \mathrm{D}$ heterojunction(s) responsible for the conductance oscillations. Interestingly, this behaviour was systematically observed on several devices with channel lengths up to $7 \mu \mathrm{m}$, far away from the confinement condition, ${ }^{[46]}$ which pinpoint the effect to be related to clusters. A careful analysis of the conductance dependency on the source-drain and back gate voltages, together with Coulomb blockade modelling based on appropriate equivalent circuit, are detailed in the following part of our article (see Figure 4) in order to access the specific electrical parameters of our $0 \mathrm{D} / 2 \mathrm{D}$ devices.

To get deeper insight into the transport processes of our devices, we performed electronic transport spectroscopy on a device in Twin configuration at $1.5 \mathrm{~K}$ at doping levels far beyond the gate voltage threshold. We provide in Supporting Information, four terminals measurements performed on this device revealing a metal to insulator transition ${ }^{[47]}$ in this gate voltage range (see Supporting Information, Figure S4). This indicates that the $\mathrm{MoS}_{2}$ channel has likely undergone a degenerated state promoted by the higher electrostatic doping level, and that the 2D channel drives charges efficiently in between the two contacts.

Figure 4a shows the intensity colormap of the conductance $\mathrm{G}$ measured with respect to gate voltage (Vg) applied through both $\mathrm{MoS}_{2}$ and silicon oxide spacer, and potential applied between source and drain electrodes (Vsd). The conductance map highlights a succession of isolated Coulomb diamonds in our $0 \mathrm{D} / 2 \mathrm{D}$ heterostructures. This supports that despite the thousands of nanoparticles potentially available in the device, only a few - and ultimately a single one effectively contribute to the charge transport through the device as previously reported for two terminals graphene based $0 \mathrm{D} / 2 \mathrm{D}$ devices. ${ }^{[14,22]}$ This further confirms that carriers tunnel 
through the cluster under Coulomb blockade regime, with a negligible contribution from direct tunnelling through the alumina tunnel barrier. In addition, Figure 4a displays on top panel typical $\mathrm{G}(\mathrm{Vg})$ curve extracted from the conductance map at $\mathrm{Vsd}=0 \mathrm{mV}$. The $\mathrm{G}(\mathrm{Vg})$ shows typical CB oscillations with respect to gate voltage. An increase of $\mathrm{G}$ intensity with $\mathrm{Vg}$ is also observed and could be originated from an external influence on the SET.

We now focus on the progressive reduction of the Coulomb diamonds (Figure 4a) with increasing gate voltage. Gate modulation of Coulomb diamonds amplitude has been previously reported on high quality semiconducting nanostructures. This was translating a change upon gating of the dot charging energy, either due to a modulation of the dot electrostatic confinement profile, or to interplay of the Coulomb interaction and additional quantum effects associated with very low electron number on the dot. ${ }^{[49,50]}$ In our 0D/2D heterostructure, such effects are unlikely. First because the nanoclusters involved are metallic and relatively large in size $\sim 10$ $\mathrm{nm}$, and also because the Coulomb oscillations were observed even with several $\mu \mathrm{m}$ (long and wide) MoS2 channels and up to relatively high temperature of several tenth of Kelvin. Here, we ascribe our unconventional backgate modulation of the metallic clusters Coulomb blockade features to the gate controlled doping of the $\mathrm{MoS}_{2}$ channel. In order to investigate the influence of the $\mathrm{MoS}_{2}$ channel on Coulomb Blockade in our Al clusters, we performed Coulomb Diamonds maps simulations of our devices in series with the channel in the framework of the Coulomb Blockade Orthodox theory. ${ }^{[50,51]}$ Figure $4 \mathrm{~b}$ shows the result of the Coulomb diamonds map scaling from 59 to $67 \mathrm{~V}$ for $\mathrm{Vg}$, and between -20 to $20 \mathrm{mV}$ for $\mathrm{Vsd}$. Clear CB diamonds are visible with gap size vanishing when increasing gate voltage. In these simulations, we implemented the gate dependent $\mathrm{MoS}_{2}$ influence on both the dot-to-channel resistance $\boldsymbol{R}_{2}(\mathrm{Vg})$ and the channel resistance $\boldsymbol{R}_{\text {Channel }}(\mathrm{Vg})$ itself (Figure 4c). While $\boldsymbol{R}_{2}(\mathrm{Vg})$ directly impacts the Coulomb blockade behavior of the dot, $\boldsymbol{R}_{\text {Channel }}(\mathrm{Vg})$ effectively weight the fraction of the source-drain potential directly applied to the dot. The gate voltage dependency of the $\mathrm{MoS}_{2}$ 
channel resistance $\boldsymbol{R}_{\text {Channel }}(\mathrm{Vg})$ is separately measured experimentally from four points geometry measurements and follow a quasi linear dependence from 20 to $10 \mathrm{M} \Omega$ when increasing gate voltage from 59 to $67 \mathrm{~V}$. Figure $4 \mathrm{~b}$ shows the best agreement corresponding to the simulation of a single Coulomb dot with the following resistance and capacitance for top source-to-dot (1) and bottom dot-to-channel (2) tunnel barriers $\left(\boldsymbol{R}_{1}=7 \mathrm{M} \Omega, \boldsymbol{C}_{\boldsymbol{1}}=12 \mathrm{aF}, \boldsymbol{R}_{2}=5\right.$ $\mathrm{M} \Omega, \boldsymbol{C}_{2}=8 \mathrm{aF}$ ) in series with the channel resistance $\boldsymbol{R}_{\text {Channel }}(\mathrm{Vg})$ (scheme in Figure 4c). The number of diamonds, the size of their gaps and their progressive fading with gate voltage are reproduced with accuracy. The total capacitance gives a charging energy of $\mathrm{E}_{\mathrm{C}}=e^{2} / 2 C_{\Sigma}=4$ $\mathrm{meV}$ for the nanoparticle. The surface extracted from the capacitance corresponds to a nanoparticle of approximately $10 \mathrm{~nm}$ in diameter, which is in good agreement with the upper side of the Al nanoparticle size distribution (Figure 2) where the presence of a metal core is promoted. ${ }^{[14,52]}$ While this $\boldsymbol{R}_{\text {Channel }}$ dependence can explain a slow decrease in the diamond size, this is not enough to account for the vanishing Coulomb diamond. Indeed, in order to explain this decrease one has to take into account also the gate dependence of the dot to $\mathrm{MoS}_{2}$ resistance $\boldsymbol{R}_{2}$ related to the doping of $\mathrm{MoS}_{2} \cdot{ }^{[53]}$ In the simulation, the dot to $\mathrm{MoS}_{2}$ resistance is chosen to follow the dependence of $\boldsymbol{R}_{\text {Channel }}(\mathrm{Vg})$. While $\boldsymbol{R}_{\boldsymbol{I}}$ (top metal contact to dot resistance) is kept constant $\boldsymbol{R}_{\mathbf{2}}$ is accounted for with a linear behavior varying in the range of 5 to $2.5 \mathrm{M} \Omega$. This highlights that the doping of the channel modulates as well the electron transport characteristics of the SET contrary to all metallic based SETs. ${ }^{[22,50,51,54-56]}$

Moreover, the single electron features are observed to be preserved up to relatively high temperature. Figure 5 presents $\log$ scale $\operatorname{Isd}(\mathrm{Vg})$ characteristics of the device for different temperatures. One observes a decrease of the threshold voltage $\mathrm{V}_{T H}$ and an increase of the conductance with temperature, which are additional characteristic of the semiconductor $\mathrm{MoS}_{2}$ channel. Furthermore, the Coulomb oscillations remain visible up to $50 \mathrm{~K}$. As the temperature 
increases, conductance peaks get broader, until they progressively vanish. In addition, the conductance minima of different peaks increase much faster than the corresponding maxima resulting in plateau like oscillations at high temperatures when both quantities get closer. ${ }^{[57,58]}$ This is noticeable at the temperature range of $20 \mathrm{~K}$ to $50 \mathrm{~K}$. Above, the minima become progressively larger than the maxima resulting in a vanishing of oscillations around $100 \mathrm{~K}$, corresponding to a thermal energy in good agreement with the blockade energy of the clusters in the range of $\sim 4 \mathrm{meV}$. These results are encouraging evidence of the high temperature operability of the 0D/2D SET. It motivates for further studies on the nanoclusters assembly growth mechanisms, that may enable a better control on their structural properties, for example by fine adjustment of the nominal thickness of the deposited Al layer as well as of the temperature of the substrate during vacuum deposition. ${ }^{[60]}$ Indeed, previous work demonstrated that optimizing the size distribution properties of a $2 \mathrm{D}$ assembly of $0 \mathrm{D}$ clusters inserted into a tunnel junction, was a successful strategy to achieve room temperature Coulomb blockade features. ${ }^{[61]}$ Hence, the demonstration of $\mathrm{v}$-SET operating at room temperature appears as a realistic goal within the experimental scope.

\section{Conclusion}

In conclusion, we have demonstrated the fabrication and the transport properties of a vertical single electron transistor based on 0D/2D van der Waals heterostructure. Physical-chemical analysis and modelling demonstrate that core(metal)-shell(oxide) nanoclusters can be grown over $\mathrm{MoS}_{2} 2 \mathrm{D}$ template when depositing an ultrathin aluminium based layer followed by controlled oxidation in air. This $0 \mathrm{D} / 2 \mathrm{D}$ heterostructure is then investigated as a platform to develop a vertical single electron transistor device taking advantage of the dual electric functionality of the 2D MoS2 template than can be used both as an electrode to drive charges in and out from the Coulomb island and as a dielectric layer enabling the back gate electric 
control of the Coulomb island charge state. Our 0D/2D v-SET devices demonstrate Coulomb blockade oscillations superimposed to the intrinsic field effect characteristic of the 2D semiconductor channel preserved up to 50K. Electronic transport spectroscopy and circuit theory modelling allow for the comprehensive analysis of the 0D/2D v-SET stability diagram, and pinpoint the Coulomb diamond patterns to the $0 \mathrm{D}$ nanoclusters. Interestingly, the gate control doping of the $\mathrm{MoS}_{2}$ channel provides an additional functionality, as it can be used as a knob to modulate the electric parameters of the SET Coulomb diamond patterns, which contrast with usual behaviour of traditional SETs. This work opens doors to exploring single electron device architectures using mixed dimensional van der Waals heterostructures, taking advantage of the versatility of the 2D materials library. Multifunctional optoelectronic single electron devices could be envisioned combining the OD cluster single electron properties with the remarkable optical properties of 2D semiconductors. Spintronic devices could be also addressed taking advantage of the fascinating properties of $2 \mathrm{D}$ magnetic materials, ${ }^{[61,62]}$ implementing them as magnetic gate electrodes in magneto-Coulomb devices, taking advantage of their electrically tunable magnetic anisotropy ${ }^{[63-66]}$ and high coercive magnetic field ${ }^{[64,67,68]}$ to magnetically tune the single electron charge state, or using ferroelectric layers for neuromorphic devices. ${ }^{[69,70]}$

\section{Experimental Section}

Device fabrication: The samples were fabricated using mechanically exfoliated $\mathrm{MoS}_{2}$ on silicon/silicon dioxide $(285 \mathrm{~nm})$ substrate $\left(\mathrm{Si} / \mathrm{SiO}_{2}\right)$. The $\mathrm{MoS}_{2}$ flake was then identified first by optical contrast and confirmed by Raman using a laser photon source with energy of 2.33 $\mathrm{eV}$. The electrodes are patterned by means of electron-beam lithography using a bilayer spin coated PMMA (poly(methyl methacrylate)) resist and developed in 1:3 MIBK-IPA (methyl isobutyl ketone-isopropyl) at $25{ }^{\circ} \mathrm{C}$. In the pre-patterned electrodes on exfoliated $\mathrm{MoS}_{2}$, an 
ultra-thin aluminium film of $1.7 \mathrm{~nm}$ is deposited using electron beam evaporation. The sample is then taken out of the vacuum chamber to air for oxidation. This results in $2 \mathrm{D}-0 \mathrm{D}$ heterostructure described in the main text. After the oxidation, the sample is then reintroduced into the deposition chamber and a top Co electrode is deposited of $40 \mathrm{~nm}$ thick cobalt layer capped by $10 \mathrm{~nm}$ gold followed by lift-off in acetone. A last e-beam lithography step is then performed to pattern microscale $\mathrm{Au}$ interconnects to the Co leads and extra Au electrodes as detailed in the main text.

Transport Measurements: Low-temperature electrical measurements were carried out in $\mathrm{He}$ flow cryostats of $1.5 \mathrm{~K}$ base temperature. A high precision source meter K2634B and a K2182A nanovoltmeter were used for data recording.

Sample and surface preparation and measurements for electron photoemission analysis : We have prepared clean MoS2 surface following M. D. Siao et al.. ${ }^{[71]}$ The sample were cleaved under vacuum at $10^{-8}$ mbar using a scotch tape. This procedure removes a part of the oxide but not completely. Then we perform an annealing at a maximum temperature of $300^{\circ} \mathrm{C}$ in Ultra High Vacuum for a long time to remove residual contamination (carbon/oxygen), but avoiding a transition of the semi conductive $2 \mathrm{H}$ MoS2 to $1 \mathrm{~T}$ which was observed for annealing temperature exceeding $400^{\circ} \mathrm{C}$ and is mainly attributed to a desorption of sulfur atoms. The $\mathrm{Al}$ deposition was done using a Knudsen cell operating at base pressure (under $10^{-9} \mathrm{mbar}$ ) and monitored by a quartz-microbalance.

STEM and EELS Analysis: The STEM investigations were performed on a JEOL 2100F TEM/STEM electron microscope equipped with a Cs probe corrector and a GIF Tridiem Filter. A $0.1 \mathrm{~nm}$ probe was used for all STEM analysis, while the inner and outer angles used for the ADF STEM imaging were 52 and $140 \mathrm{mrad}$, respectively. For the EELS analysis, the oxygen and aluminium scan profiles were extracted from the EELS spectra at O-K edge and Al-L2,3, respectively. 
X-ray Photoelectron Spectroscopy Measurements: XPS analysis was carried out on a hemispherical electron analyser (Scienta R3000) equipped with a monochromatic Al-Ka X-ray source $(\mathrm{h} v=1468.6 \mathrm{eV})$. The measurements were performed at $60^{\circ}$ from the sample normal.

\section{Supporting Information}

Supporting Information is available from the Wiley Online Library or from the author. It includes additional information regarding : 1. Physical-chemical analysis and modelling of the oxidation mechanism of the $\mathrm{Al}$ based nanoclusters deposited over $\mathrm{MoS}_{2}$ (XPS, ARPES, DFT). 2. Four points measurements of $\mathrm{MoS}_{2}$ channel and the metal-insulator transition.

\section{Acknowledgements}

We acknowledge the use of clean-room facilities from the STnano platform, and Fabien Chevrier for assistance with cryogenics. This work is supported by a public grant overseen by the French National Research Agency (ANR) through projects MIXES (grant ANR-19-CE090028), COPIN (ANR-19-CE24-0022), STEM2D (ANR-19-CE24-0015), FLAGERA 2019 SOgraphMEM (ANR-19-GRFI-0001-07), and as part of the "Investissements d'Avenir" program (Labex NIE, ANR-11-LABX-0058, Labex Nanosaclay, ANR-10-LABX-0035, and ANR-10-IDEX-0002-02). This work is supported by a public grant overseen by European Commission through H2020 Future and Emerging Technologies Graphene Flagship (Grants Core2 No. 785219 and Core3 No. 881603). We also acknowledge the JSPS core-to-core program. J.-F. D. and S.B. thank the Institut Universitaire de France (IUF) for financial support. Received: ((will be filled in by the editorial staff)) Revised: ((will be filled in by the editorial staff)) Published online: ((will be filled in by the editorial staff))

\section{References}


[1] K. S. Novoselov, A. Mishchenko, A. Carvalho, A. H. Castro Neto, Science 2016, 353, 6298.

[2] P. Ajayan, P. Kim, K. Banerjee, Phys. Today 2016, 69, 38.

[3] S. Luryi, Appl. Phys. Lett. 1988, 52, 501.

[4] T. Tian, P. Rice, E. J. G. Santos, C. J. Shih, Nano Lett. 2016, 16, 5044.

[5] S. Das, J. Appenzeller, Phys. Status Solidi - Rapid Res. Lett. 2013, 7, 268.

[6] S. Das, J. Appenzeller, Nano Lett. 2013, 13, 3396.

[7] D. Jariwala, T. J. Marks, M. C. Hersam, Nat. Mater. 2017, 16, 170.

[8] D. Jariwala, V. K. Sangwan, C.-C. Wu, P. L. Prabhumirashi, M. L. Geier, T. J. Marks, L. J. Lauhon, M. C. Hersam, Proc. Natl. Acad. Sci. 2013, 110, 18076.

[9] D. Kufer, I. Nikitskiy, T. Lasanta, G. Navickaite, F. H. L. Koppens, G. Konstantatos, Adv. Mater. 2015, 27, 176.

[10] U. N. Noumbé, C. Gréboval, C. Livache, A. Chu, H. Majjad, L. E. Parra López, L. D. N. Mouafo, B. Doudin, S. Berciaud, J. Chaste, A. Ouerghi, E. Lhuillier, J. F. Dayen, ACS Nano 2020, 14, 4567.

[11] A. Mahmood, C. S. Yang, S. Jang, L. Routaboul, H. Chang, A. Ghisolfi, P. Braunstein, L. Bernard, T. Verduci, J. F. Dayen, P. Samorì, J. O. Lee, B. Doudin, Nanoscale 2019, $11,19705$.

[12] J. F. Dayen, S. J. Ray, O. Karis, I. J. Vera-Marun, M. V. Kamalakar, Appl. Phys. Rev. 2020, 7, 011303.

[13] M. Piquemal-Banci, R. Galceran, M.-B. Martin, F. Godel, A. Anane, F. Petroff, B. Dlubak, P. Seneor, J. Phys. D. Appl. Phys. 2017, 50, 203002.

[14] L. D. N. Mouafo, F. Godel, G. Melinte, S. Hajjar-Garreau, H. Majjad, B. Dlubak, O. Ersen, B. Doudin, L. Simon, P. Seneor, J.-F. Dayen, Adv. Mater. 2018, 1802478.

[15] M. Piquemal-Banci, R. Galceran, F. Godel, S. Caneva, M.-B. Martin, R. S. Weatherup, 
P. R. Kidambi, K. Bouzehouane, S. Xavier, A. Anane, F. Petroff, A. Fert, S. M.-M.

Dubois, J.-C. Charlier, J. Robertson, S. Hofmann, B. Dlubak, P. Seneor, ACS Nano 2018, $12,4712$.

[16] V. Zatko, M. Galbiati, S. M. M. Dubois, M. Och, P. Palczynski, C. Mattevi, P. Brus, O. Bezencenet, M. B. Martin, B. Servet, J. C. Charlier, F. Godel, A. Vecchiola, K. Bouzehouane, S. Collin, F. Petroff, B. Dlubak, P. Seneor, ACS Nano 2019, 13, 14468.

[17] F. Godel, M. Venkata Kamalakar, B. Doudin, Y. Henry, D. Halley, J.-F. Dayen, Appl. Phys. Lett. 2014, 105, 152407.

[18] G. Song, M. Ranjbar, D. R. Daughton, R. A. Kiehl, Nano Lett. 2019, 19, 7112.

[19] A. Allain, Z. Han, V. Bouchiat, Nat. Mater. 2012, 11, 590.

[20] B. M. Kessler, Ç. Ö. Girit, A. Zettl, V. Bouchiat, Phys. Rev. Lett. 2010, 104, 047001.

[21] K. Song, D. Soriano, A. W. Cummings, R. Robles, P. Ordejón, S. Roche, Nano Lett. 2018, 18, 2033.

[22] F. Godel, L. D. N. Mouafo, G. Froehlicher, B. Doudin, S. Berciaud, Y. Henry, J.-F. Dayen, D. Halley, Adv. Mater. 2017, 29, 1604837.

[23] S. Linas, F. Jean, T. Zhou, C. Albin, G. Renaud, L. Bardotti, F. Tournus, Sci. Rep. 2015, 5,1

[24] P. Capiod, L. Bardotti, A. Tamion, O. Boisron, C. Albin, V. Dupuis, G. Renaud, P. Ohresser, F. Tournus, Phys. Rev. Lett. 2019, 122, 106802.

[25] B. Wang, B. Yoon, M. König, Y. Fukamori, F. Esch, U. Heiz, U. Landman, Nano Lett. 2012, 12, 5907.

[26] Y. Liu, N. O. Weiss, X. Duan, H.-C. Cheng, Y. Huang, X. Duan, Nat. Rev. Mater. 2016, $1,16042$.

[27] G. Kim, S.-H. Jhi, ACS Nano 2011, 5, 805.

[28] R. Baltic, M. Pivetta, F. Donati, C. Wäckerlin, A. Singha, J. Dreiser, S. Rusponi, H. 
Brune, Nano Lett. 2016, 16, 7610.

[29] D. C. Elias, R. R. Nair, T. M. G. Mohiuddin, S. V. Morozov, P. Blake, M. P. Halsall, A. C. Ferrari, D. W. Boukhvalov, M. I. Katsnelson, A. K. Geim, K. S. Novoselov, Science. 2009, 323, 610.

[30] U. Chandni, K. Watanabe, T. Taniguchi, J. P. Eisenstein, Nano Lett. 2016, 16, 12 , 7982.

[31] R. Pisoni, Z. Lei, P. Back, M. Eich, H. Overweg, Y. Lee, K. Watanabe, T. Taniguchi, T. Ihn, K. Ensslin, Appl. Phys. Lett. 2018, 112, 123101.

[32] K. Wang, K. De Greve, L. A. Jauregui, A. Sushko, A. High, Y. Zhou, G. Scuri, T. Taniguchi, K. Watanabe, M. D. Lukin, H. Park, P. Kim, Nat. Nanotechnol. 2018, 13, 128.

[33] D. Bischoff, M. Eich, A. Varlet, P. Simonet, H. C. Overweg, K. Ensslin, T. Ihn, Mater. Today 2016, 19, 375 .

[34] K. S. Novoselov, A. K. Geim, S. V Morozov, D. Jiang, Y. Zhang, S. V Dubonos, I. V Grigorieva, A. A. Firsov, Science. 2004, 306, 666.

[35] S. Y. Chen, C. Zheng, M. S. Fuhrer, J. Yan, Nano Lett. 2015, 15, 4, 2526.

[36] P. Soubelet, A. E. Bruchhausen, A. Fainstein, K. Nogajewski, C. Faugeras, Phys. Rev. B 2016, 93, 155407.

[37] C. Lee, H. Yan, L. E. Brus, T. F. Heinz, J. Hone, S. Ryu, ACS Nano 2010, 4, 2695.

[38] X. Shi, G. Dong, M. Fang, F. Wang, H. Lin, W. C. Yen, K. S. Chan, Y. L. Chueh, J. C. Ho, J. Mater. Chem. C 2014, 2, 5417.

[39] J. P. Lewis, P. Jelínek, J. Ortega, A. A. Demkov, D. G. Trabada, B. Haycock, H. Wang, G. Adams, J. K. Tomfohr, E. Abad, H. Wang, D. A. Drabold, Phys. status solidi 2011, 248, 1989.

[40] M. A. Basanta, Y. J. Dappe, P. Jelínek, J. Ortega, Comput. Mater. Sci. 2007, 39, 759. 
[41] S. Dubey, S. Lisi, G. Nayak, F. Herziger, V. D. Nguyen, T. Le Quang, V. Cherkez, C. González, Y. J. Dappe, K. Watanabe, T. Taniguchi, L. Magaud, P. Mallet, J. Y. Veuillen, R. Arenal, L. Marty, J. Renard, N. Bendiab, J. Coraux, V. Bouchiat, ACS Nano 2017, 11, 11206.

[42] L. D. N. Mouafo, F. Godel, G. Froehlicher, S. Berciaud, B. Doudin, M. Venkata Kamalakar, J.-F. Dayen, 2D Mater. 2017, 4, 015037.

[43] U. N. Noumbé, C. Gréboval, C. Livache, T. Brule, B. Doudin, A. Ouerghi, E. Lhuillier, J. F. Dayen, Adv. Funct. Mater. 2019, 29, 1902723.

[44] A. Dankert, M. V. Kamalakar, A. Wajid, R. S. S. Patel, S. P. P. Dash, Nano Res. 2014, $8,1357$.

[45] J.-R. Chen, P. M. Odenthal, A. G. Swartz, G. C. Floyd, H. Wen, K. Y. Luo, R. K. Kawakami, Nano Lett. 2013, 13, 7, 3106.

[46] K. Lee, G. Kulkarni, Z. Zhong, Nanoscale 2016, 8, 7755.

[47] B. Radisavljevic, A. Kis, Nat. Mater. 2013, 12, 815.

[48] S. J. Shin, C. S. Jung, B. J. Park, T. K. Yoon, J. J. Lee, S. J. Kim, J. B. Choi, Y. Takahashi, D. G. Hasko, Appl. Phys. Lett. 2010, 97, 103101.

[49] A. T. Tilke, F. C. Simmel, R. H. Blick, H. Lorenz, J. P. Kotthaus, Prog. Quantum Electron. 2001, 25, 97.

[50] K. K. Likharev, Proc. IEEE 1999, 87, 606.

[51] A. Bernand-Mantel, P. Seneor, K. Bouzehouane, S. Fusil, C. Deranlot, F. Petroff, A. Fert, Nat. Phys. 2009, 5, 920.

[52] G. Maidecchi, C. V. Duc, R. Buzio, A. Gerbi, G. Gemme, M. Canepa, F. Bisio, J. Phys. Chem. C 2015, 119, 26719.

[53] A. Allain, J. Kang, K. Banerjee, A. Kis, Nat. Mater. 2015, 14, 1195.

[54] K. I. Bolotin, F. Kuemmeth, A. N. Pasupathy, D. C. Ralph, Appl. Phys. Lett. 2004, 84, 
3154.

[55] K. S. Makarenko, Z. Liu, M. P. de Jong, F. A. Zwanenburg, J. Huskens, W. G. van der Wiel, Adv. Mater. 2017, 29, 42, 1702920.

[56] A. V. Danilov, D. S. Golubev, S. E. Kubatkin, Phys. Rev. B - Condens. Matter Mater. Phys. 2002, 65, 125312.

[57] H. van Houten, C.W.J. Beenakker and A.A.M. Staring, in: Single Charge Tunneling, Eds. H. Grabert and M.H. Devoret, NATO ASI Series B (Plenum, New York).

[58] A. I. Yakimov, V. A. Markov, A. V Dvurechenskii, O. P. Pchelyakov, J. Phys. Condens. Matter 1994, 6, 2573.

[59] A. Baskaran, P. Smereka, J. Appl. Phys. 2012, 111, 044321.

[60] N. Lidgi-Guigui, P. Seneor, F. Nguyen Van Dau, A. Friederich, A. Vaur̀s, C. Deranlot, Appl. Phys. Lett. 2007, 90, 233101.

[61] K. F. Mak, J. Shan, D. C. Ralph, Nat. Rev. Phys. 2019, 1, 646.

[62] M. Gibertini, M. Koperski, A. F. Morpurgo, K. S. Novoselov, Nat. Nanotechnol. 2019, $14,408$.

[63] T. Maruyama, Y. Shiota, T. Nozaki, K. Ohta, N. Toda, M. Mizuguchi, A. A. Tulapurkar, T. Shinjo, M. Shiraishi, S. Mizukami, Y. Ando, Y. Suzuki, Nat. Nanotechnol. 2009, 4, 158.

[64] B. Huang, G. Clark, D. R. Klein, D. MacNeill, E. Navarro-Moratalla, K. L. Seyler, N. Wilson, M. A. McGuire, D. H. Cobden, D. Xiao, W. Yao, P. Jarillo-Herrero, X. Xu, Nat. Nanotechnol. 2018, 13, 544.

[65] S. Jiang, L. Li, Z. Wang, K. F. Mak, J. Shan, Nat. Nanotechnol. 2018, 13, 549.

[66] J. Kim, K. W. Kim, B. Kim, C. J. Kang, D. Shin, S. H. Lee, B. C. Min, N. Park, Nano Lett. 2020, 20, 929.

[67] Z. Fei, B. Huang, P. Malinowski, W. Wang, T. Song, J. Sanchez, W. Yao, D. Xiao, X. 
Zhu, A. May, W. Wu, D. Cobden, J.-H. Chu, X. Xu, Nat. Mater. 2018, 17, 778.

[68] C. Gong, L. Li, Z. Li, H. Ji, A. Stern, Y. Xia, T. Cao, W. Bao, C. Wang, Y. Wang, Z. Q. Qiu, R. J. Cava, S. G. Louie, J. Xia, X. Zhang, Nature 2017, 546, 265.

[69] V. K. Sangwan, M. C. Hersam, Nat. Nanotechnol. 2020, 15, 517.

[70] S. Pecqueur, D. Vuillaume, F. Alibart, J. Appl. Phys. 2018, 124, 151902.

[71] M. D. Siao, W. C. Shen, R. S. Chen, Z. W. Chang, M. C. Shih, Y. P. Chiu, C. M. Cheng, Nat. Commun. 2018, 9, 1. 

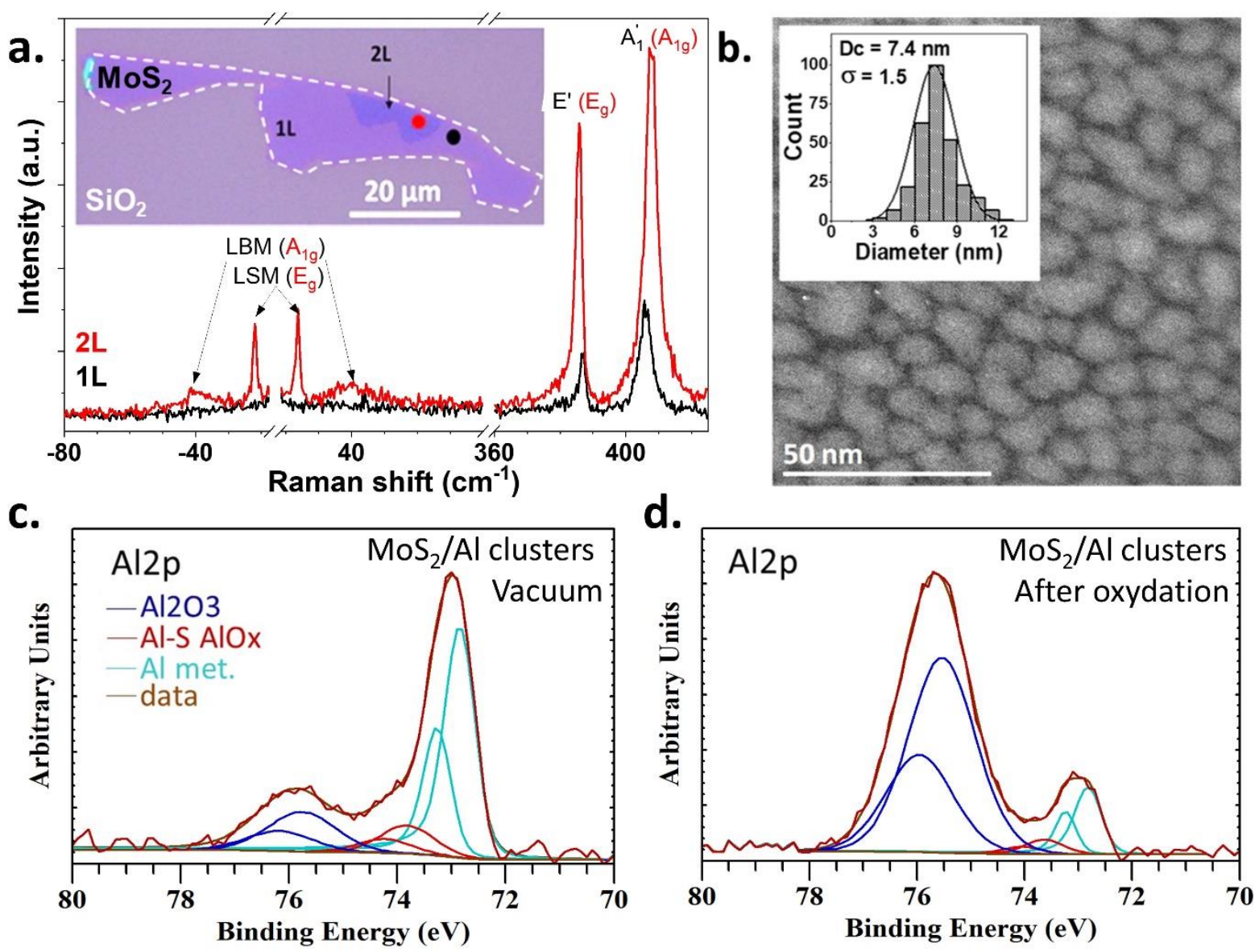

Figure 1: a. Raman spectra recorded at the mono (1L) and bilayer $(2 L) \mathrm{MoS}_{2}$ regions indicated respectively the black and red dot on the inserted optical micrograph of the flake, with labelling of their Raman modes. b. Scanning transmission electron microscopy bright field image of the ultrathin oxidized Al layer deposited on the surface of a MoS $\mathrm{S}_{2}$ flake transferred on a carbon TEM grid. Inset : the corresponding histogram of the clusters' diameter $D$, fitted with a Gaussian curve centered at DC $=7.4 \mathrm{~nm}$ with a standard deviation $\sigma$ of $1.5 \mathrm{~nm}$ (20\%). c. and d. show respectively the X-ray photoelectron measurement of the Al2p spectra (c) after deposition of the Al layer on $\mathrm{MoS}_{2}$ in Ultra High Vacuum (UHV) conditions and (d) after 60 min of oxidation time in 70 mbar oxygen pressure. After oxidation, in addition to signatures of $\mathrm{Al}_{2} \mathrm{O}_{3}$ and Al-S AlOx interface, the persistence of metallic Al ("Al met.") is observed confirming further the core (metal) / shell (oxide) structure of the nanoclusters. Dual peaks of the same color correspond to the spin-orbit doublet $A l_{2 p}(3 / 2)$ and $A l_{2 p}(1 / 2)$ of the $A l_{2 p}$ core level peak, that are observed for the metallic, interfacial and oxidized sates of Al. 
a.

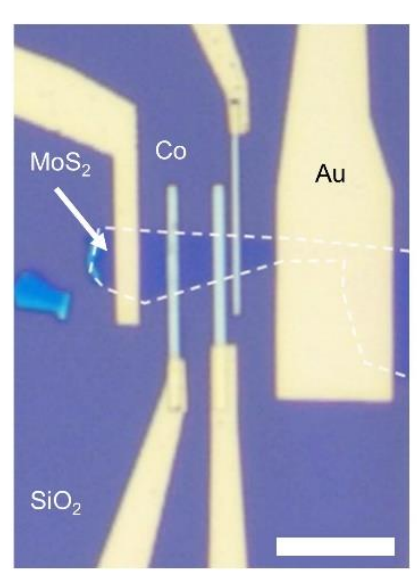

b. Single Cell

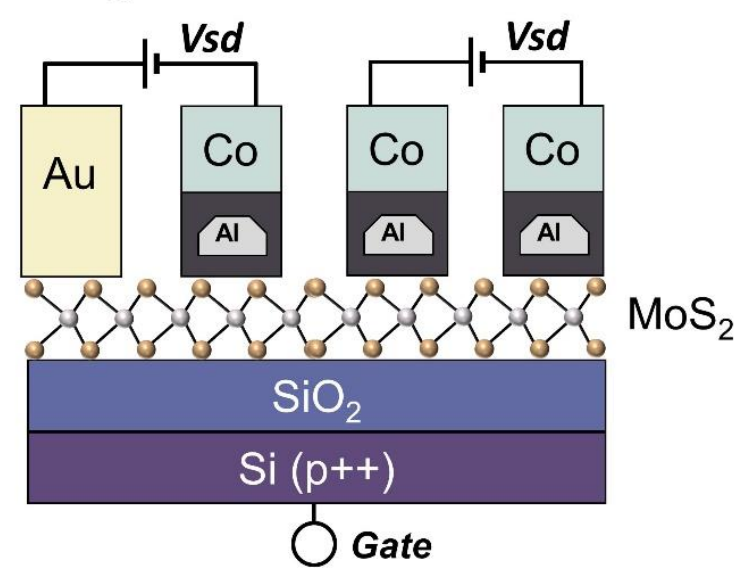

Figure 2: a. Optical micrograph of the device, the scale bar is $10 \mu \mathrm{m} . b$. Schematic representation of the vertical-SET device made of MoS flake over $\mathrm{Si}_{2}(290 \mathrm{~nm}) /$ Si substrate, contacted by nanoelectrodes incorporating the nanoclusters (light grey color) and by gold electrodes (in yellow). 
a.
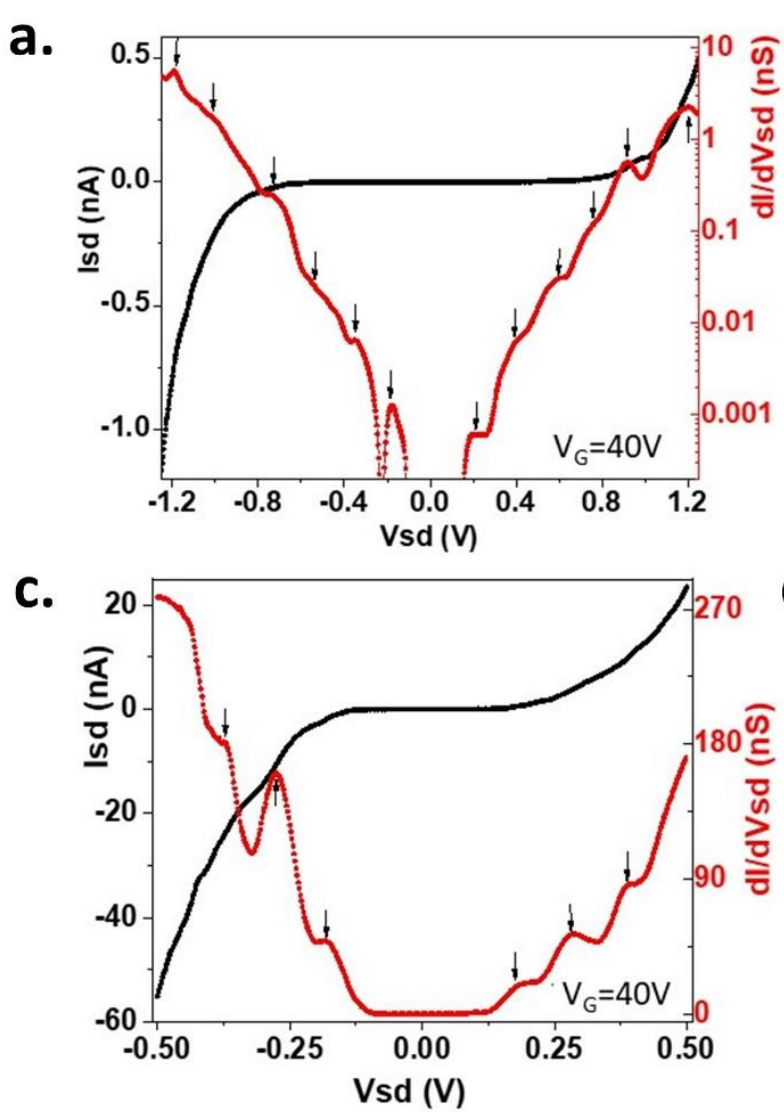
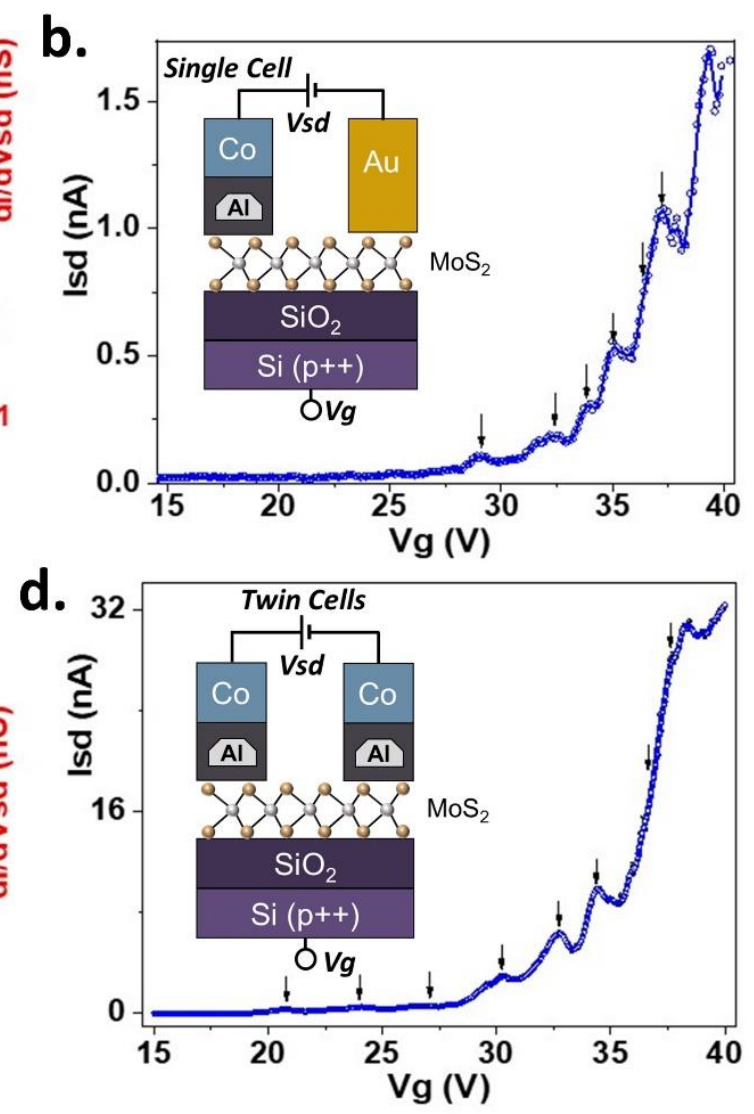

Figure 3: Electronic transport measurements at low temperature $(1.5 \mathrm{~K})$ of two representative devices in single cell $(a, b)$ and twin cells $(c, d)$ configurations. The single cell device has a channel length of $3.5 \mu \mathrm{m}$ and $a$ width of $5 \mu \mathrm{m}$, and the twin cells device a channel length of 350 $\mathrm{nm}$ and a width of $10 \mu \mathrm{m}$. a. Isd-Vsd characteristic (black curves, left axis) and the corresponding $d I / d V s d(V s d)$ curve (red curve, right axis) measured at $V_{G}=40 \mathrm{~V}$ for the single cell device. The black arrows highlight the positions of the conductance peaks. b. Isd(Vg) curve measured at $V s d=1.5 \mathrm{~V}$. The black arrows highlight the positions of the current oscillations $c$. Isd-Vsd characteristic (black curve, left axis) and the corresponding differential conductance (red curve, right axis) measured at Vg $=29 \mathrm{~V}$ on the twin cell device. $d$ ) Isd(Vg) curve measured for $V s d=230 \mathrm{mV}$. The inset highlights the low Vg region of the main curve and the current oscillations. 

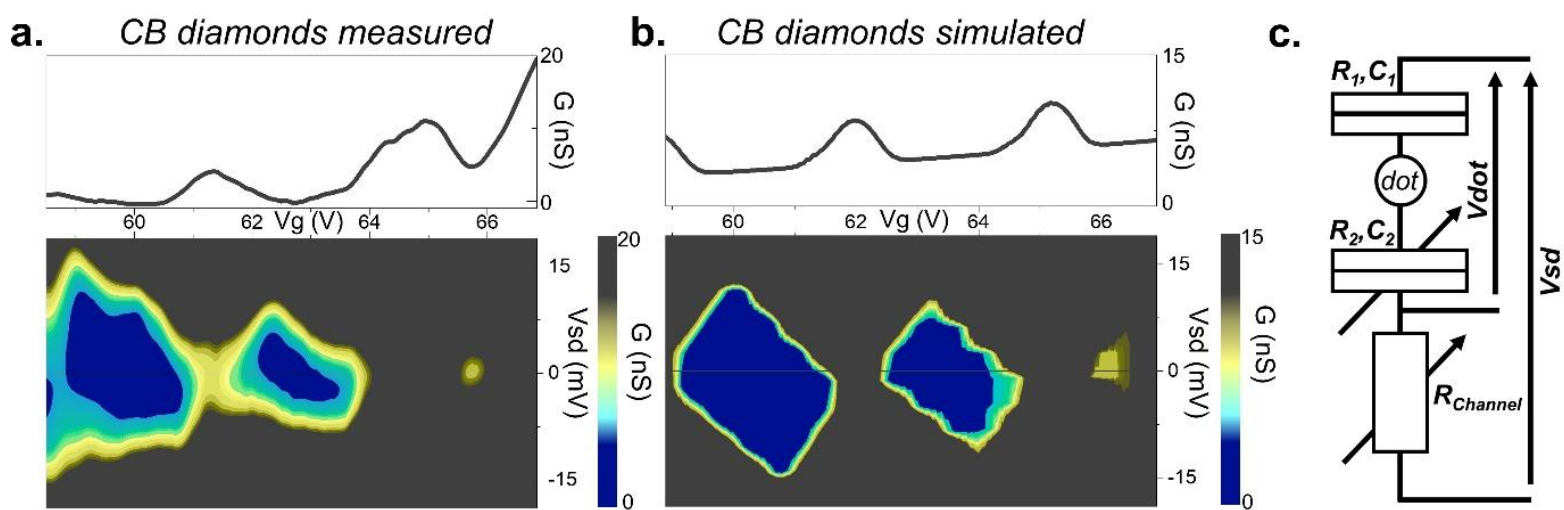

Figure 4: Vertical single electron transistor stability diagram. a. Coulomb Diamond (CB) map measured on Al nanocluster/MoS 2 device. Top panel represents the $G(V g)$ profile extracted from $G(V g, V s d)$ map at $V s d=0 \mathrm{mV}$. b. Simulated Coulomb Diamonds map using Orthodox method on single dot. Top panel represents the $G(V g)$ profile extracted from calculated $G(V g$, $V s d)$ map at $V s d=0 \mathrm{mV}$. c) Scheme of the influence of the source-drain potential (Vsd) on voltage applied to the dot (Vdot) weighting through voltage divider. 


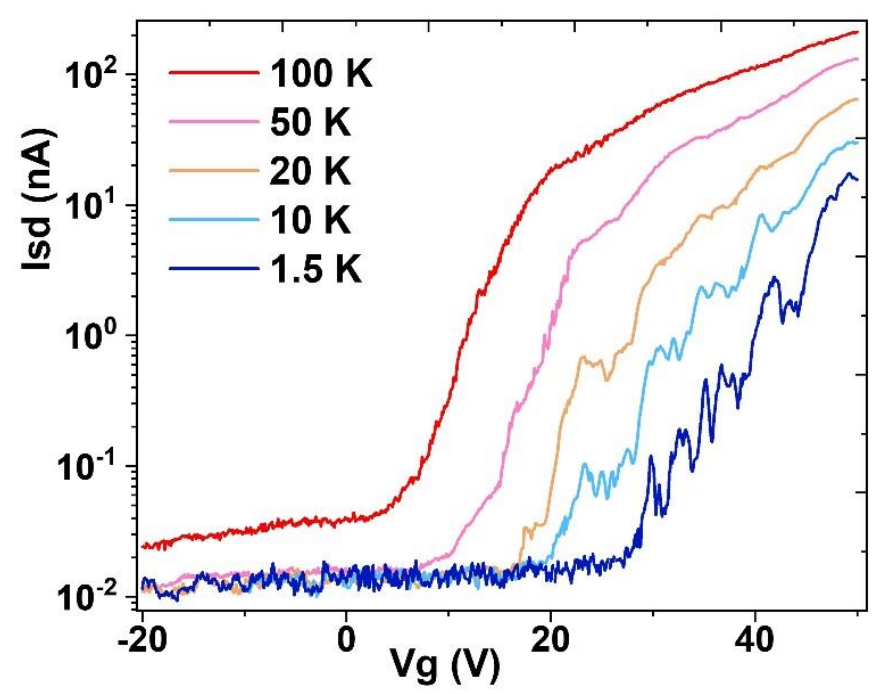

Figure 5: Temperature dependent transfers characteristics of the 0D/2D SET in Twin cells. Coulomb oscillations remained preserved up to $50 \mathrm{~K}$. 


\section{Table of contents entry:}

The concept of 0D-2D Vertical Single Electron Transistor is unveiled. It allows to combine the large Coulomb energy of nanoclusters with the electronic capabilities of a $2 \mathrm{D}$ channel that can act both as a bottom electrode and a dielectric for back gate control of the nanoclusters charge states. These findings open doors to unexplored architecture of multifunctional single electron device based on Mixed-Dimensional Heterostructures.

L.D.N. Mouafo, F. Godel, L. Simon*, Y. J. Dappe*, W. Baziz, U.N. Noumbé, E. Lorchat, M.B. Martin, S. Berciaud, B. Dlubak, B. Doudin, O. Ersen, P. Seneor and J.-F. Dayen*.

\section{D-2D Heterostructures Vertical Single Electron Transistor}
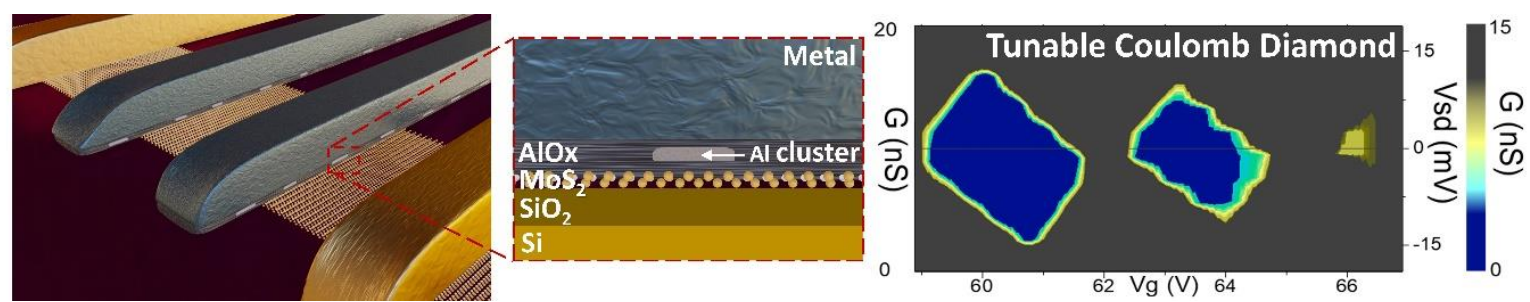
Copyright WILEY-VCH Verlag GmbH \& Co. KGaA, 69469 Weinheim, Germany, 2018.

\section{Supporting Information}

\section{D-2D Heterostructures Vertical Single Electron Transistor}

Louis Donald Notemgnou Mouafo, Florian Godel, Laurent Simon*, Yannick J. Dappe*, Waalid Baziz, Ulrich Nguétchuissi Noumbé, Etienne Lorchat, Marie-Blandine Martin, Stéphane, Berciaud, Bruno Dlubak, Bernard Doudin, Ovidiu Ersen, Pierre Seneor*, Jean-Francois Dayen*

\section{Table of contents}

1. Physico-chemical analysis and modelling of the oxidation mechanism of the Al based nanoclusters deposited over $\mathrm{MoS}_{2}$.

2. Four points measurements of $\mathrm{MoS}_{2}$ channel . .4

1. Physico-chemical analysis and modelling of the oxidation mechanism of the Al based nanoclusters deposited over $\mathrm{MoS}_{2}$.

In order to understand the nature of the aluminium oxidation process and the interface between the oxidized aluminium dots and the $\mathrm{MoS}_{2}$ substrate, we have performed systematic analysis by combining scanning tunneling microscopy and electron photoemission measurements. In a first experiment we have followed the nominal parameters used for the fabrication of the device (typically oxidation in air for $\mathrm{Al}$ deposition thickness of 1.5 to $2 \mathrm{~nm}$ ), with a control of the surface at each step of the process: the preparation of pristine $\mathrm{MoS}_{2}$, the deposition of $\mathrm{Al}$ and 
the oxidation are done with the transfer of the sample in different UHV chambers without breaking the vacuum. In our system, oxygen $\left(\mathrm{O}_{2}\right)$ exposure can be done from few Langmuir to 0.5 bar of pressure. The pressure is measured using a cold-cathode gauge which allows to follows the pressure from $5.10^{-9} \mathrm{mbar}$ to $100 \mathrm{mbar}$.

We prepare clean $\mathrm{MoS}_{2}$ surface following M. D. Siao et al. ${ }^{[1]}$. The sample is cleaved under vacuum at $10^{-8}$ mbar using a scotch tape. This procedure removes a part of the oxide but not completely. Then we perform an annealing at a maximum temperature of $300^{\circ} \mathrm{C}$ for a long time. This procedure has been determined in order to remove residual contamination (carbon/oxygen) which needs to degas the sample (overnight annealing at $5.10^{-11} \mathrm{mbar}$ ) but avoiding a transition of the semi conductive $2 \mathrm{H} \mathrm{MoS}$ to $1 \mathrm{~T}$ which was observed during annealing process for annealing temperature exceeding $400^{\circ} \mathrm{C}$ and is mainly attributed to a desorption of sulfur atoms. Al deposition was done using a Knudsen cell operating at base pressure (under $10^{-9} \mathrm{mbar}$ ), and the deposited thickness is controlled by a quartz-microbalance.

Figure $\mathbf{S} 1$ shows the core level peak $\mathrm{Al}_{2 \mathrm{P}}$ taken with a monochromatized $\mathrm{AlK} \alpha \mathrm{X}$-ray line (electron analyzer Scienta R3000 which reach $16 \mathrm{meV}$ of resolution for a pass energy of 20eV) at an emission angle normal to the sample surface, just after the deposition of $2 \mathrm{~nm}$ of $\mathrm{Al}$ at room temperature (Figure S1a) and for two subsequent stages of oxidation (Figure S1b and Figure S1c). After the oxidation of $2 \mathrm{~h} 30 \mathrm{~min}$ at $18 \mathrm{mbar}$, the Al2p core level peak does not change, and remains unchanged even after longer oxidation time at higher pressure $(2 \mathrm{~h} 30 \mathrm{~min}$ at 60 mbar).

We identify Al metallic part with the two components Al2p (3/2) (72.82 eV) and Al2p (1/2) $(73.24 \mathrm{eV})$ with a spin-orbit splitting of $0.42 \mathrm{eV}$. Just after deposition and even under UHV conditions, due to the high reactivity of $\mathrm{Al}$, we always have residual oxide component $\mathrm{AlOx}$ observed around $75.8 \mathrm{eV}$. Between this AlOx component and $\mathrm{Al}$ metallic we clearly resolve a supplementary component around a binding energy of $73.7 \mathrm{eV}$ which is attributed to an 
interfacial state between $\mathrm{MoS}_{2}$ and aluminium oxide. The nature of this component will be discussed later. In Figure S1b the oxidation leads to an increase of the AlOx intensity and a decrease of the contribution of the metallic Al ("Al met") component. For an oxidation in air with much longer oxidation time that the one used for the device fabrication (here $3 \mathrm{~h} 30$ ) we still see (Figure S1c) a residual Al metal component together with interfacial state. We conclude that this oxidation process leads to the formation of core-shell structure with a metallic core and a $\mathrm{AlOx}$ shell.

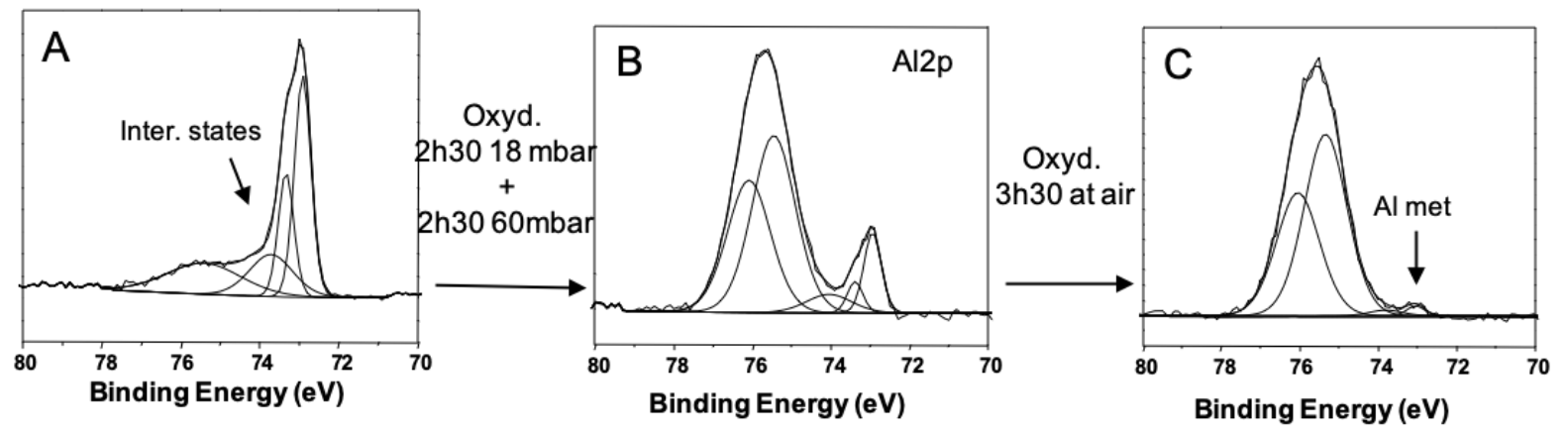

Figure S1. a-c. Al2p core level spectra taken at normal incidence. After deposition on clean $\mathrm{MoS}_{2}$ of $0.5 \mathrm{~nm} \mathrm{Al}$ at RT (a.), and for subsequent oxidation stage (b. and c.). The residual Al metallic component even after long oxidation time in air strongly suggests the formation of core-shell structure dots with a metallic core surrounded by oxidized aluminium shell.

In order to identify the origin of the interfacial state, we have studied the oxidation process of lower thicknesses i. e. $0.5 \mathrm{~nm}$.

For the pristine $\mathrm{MoS}_{2}$, the S2p and Mo3d core level spectra are shown in black in Figure S2 (Figure S2a and Figure S2d respectively). The S2p peak is easily deconvoluted by two symmetrical Gaussian-Lorentzian GL(30) with a FWHM of $0.5 \mathrm{eV}$ and a SO splitting of \pm $0.18 \mathrm{eV}$. After deposition of $0.5 \mathrm{~nm}$ of $\mathrm{Al}$, the $\mathrm{S} 2 \mathrm{p}$ core level peak shows (Figure S2b) two supplementary components both shifted by $0.4 \mathrm{eV}$ with an intensity which reach $50 \%$ of the 
initial sulfur atoms. In Figure S2c, the Mo3d core level peak before and after aluminium deposition do not change except a global energy shift of $0.1 \mathrm{eV}$ toward low binding energy and global decrease of intensity (50\%) due to the screening of a part of the $\mathrm{MoS}_{2}$ substrate by the aluminium overlayer. STM images after deposition (see insert in Figure S2b) shows flat islands with a height of up to $3 \mathrm{~nm}$. Flooding tool using SWXM software ${ }^{[2]}$ show that the islands are separated by an average distance of $18 \pm 2 \mathrm{~nm}$, and a whole volume of about $50000 \mathrm{~nm}^{3}$ that recover $46 \%$ of the surface. The volume of the island corresponds to $0.45 \mathrm{~nm}$ of an equivalent 2D Al layer, in strong accordance with the deposition thickness measured with quartz microbalance $(0.5 \mathrm{~nm})$. The two core level peaks observed in the $\mathrm{S} 2 \mathrm{p}$ spectra are then unambiguously attributed to the modification of the coordination of $\mathrm{S}$ atoms at the interface with $\mathrm{Al}$ islands.

In Figure S2c, as previously discussed, the Al2p spectra reveals the Al metallic components of the oxidized AlOx and the interfacial state. The intensity of this interfacial component increases by changing the collection angle of photoemitted electron from grazing to normal supporting the interfacial character. In the deconvolution, following G. Maidecchi et al. ${ }^{[3]}$, we used the asymmetrical Doniach-Sunjic doublet peak for the A12p 3/2 and 1/2 component, usually used for 2D metallic aluminium layer. Figure S2d shows the superimposition of the Mo3d core level peak on pristine $\mathrm{MoS}_{2}$ (black curve) and after Al deposition (red curve). As excepted, a decrease of the intensity and an energy shift of $0.1 \mathrm{eV}$ toward lower binding energy for both Mo3d 3/2 and 5/2 core level peak, can be perfectly superimposed and no supplementary component are observed, indicating that Mo atoms are not perturbated by Al. We conclude that the interfacial state is probably due to S-Al-O bonds. We notice also that it is not observed a transition from 2H to $1 \mathrm{~T}$ which would have modified both $\mathrm{S} 2 \mathrm{p}$ and $\mathrm{MoS}_{2}$ core level peak similarly. 

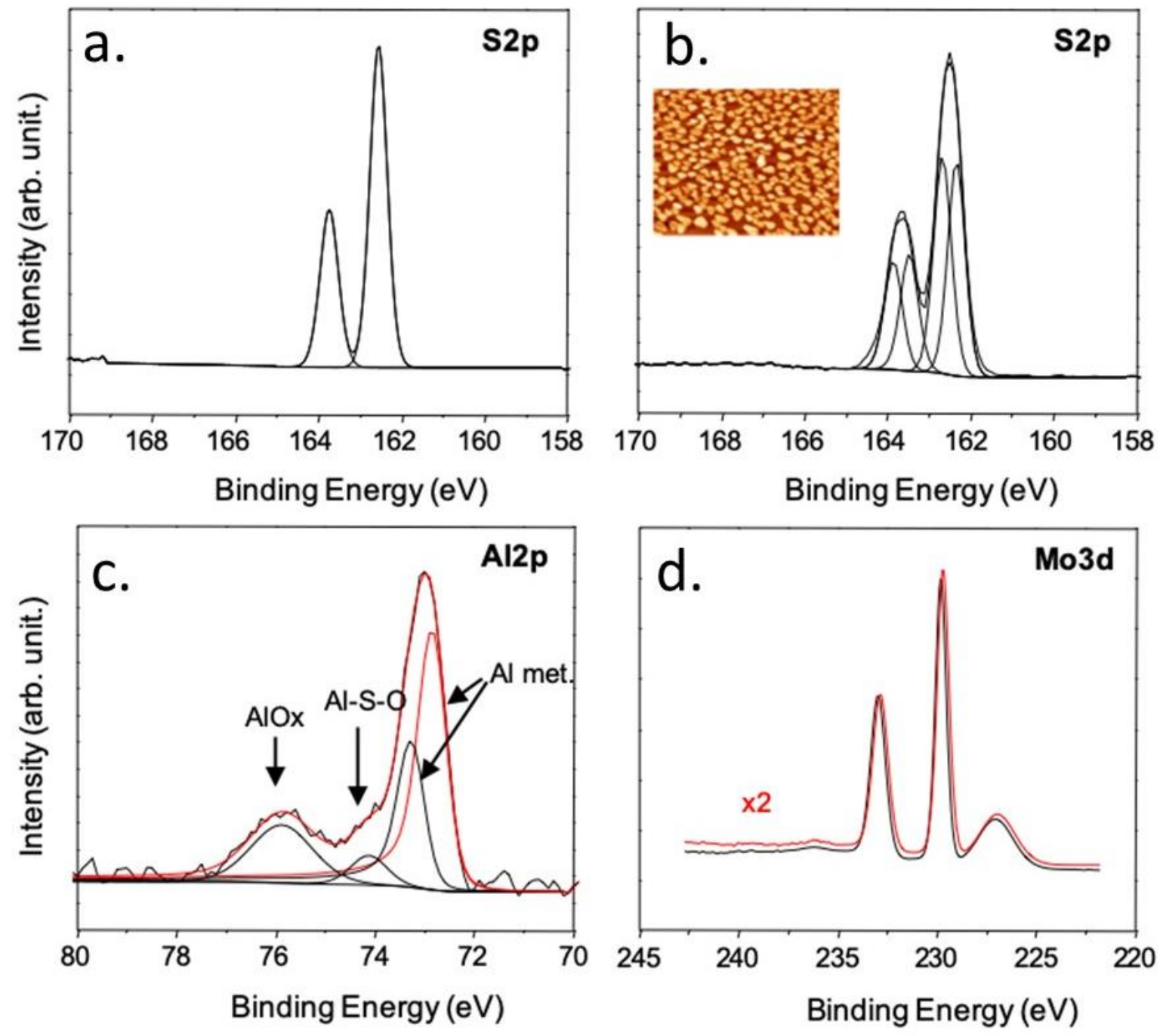

Figure S2.a. S $2 p$ core level peak on pristine $M_{o} S_{2}$. b. and c. S2p and Al2p core level peak after $0.5 \mathrm{~nm}$ Al deposition. The Insert image is $400 \times 300 \mathrm{~nm}^{2}$ STM image taken at $-2 \mathrm{~V}, 150 \mathrm{pA}$. In d., superimposed Mo3d core level peaks before (black curve) and after (red curve, intensity x2) Al deposition.

We have also probe the valence band structure by ARPES measurements before/after $\mathrm{Al}$ deposition and after subsequent oxidation (Figure S3a), while depositing of a lower quantity of $\mathrm{Al}(0.25 \mathrm{~nm})$. As the UV photoelectron is highly sensitive to the surface we avoid to bury the interface feature in the valence band spectra. The spectra are taken at normal incidence (Gamma point). Valence band width of $6 \mathrm{eV}$ is the hybridization of Mo2d and S3p. The top of the max 
at gamma is a mix of Mo $4 \mathrm{dz}^{2}$ and S $3 \mathrm{p}_{\mathrm{z}}$. The marked small feature (see dot line) has a strong Mo4dz2 character ${ }^{[4]}$ while the apex of the band structure at Gamma reveal much more the Spz valence band orbital. After Al deposition we observe an energy shift of around $0.13 \mathrm{eV}$ toward $\mathrm{E}_{\mathrm{f}}$ and a feature around $-1.3 \mathrm{eV}$. We notice that this feature is particularly marked and resolved at the apex of the valence band spectrum at gamma.
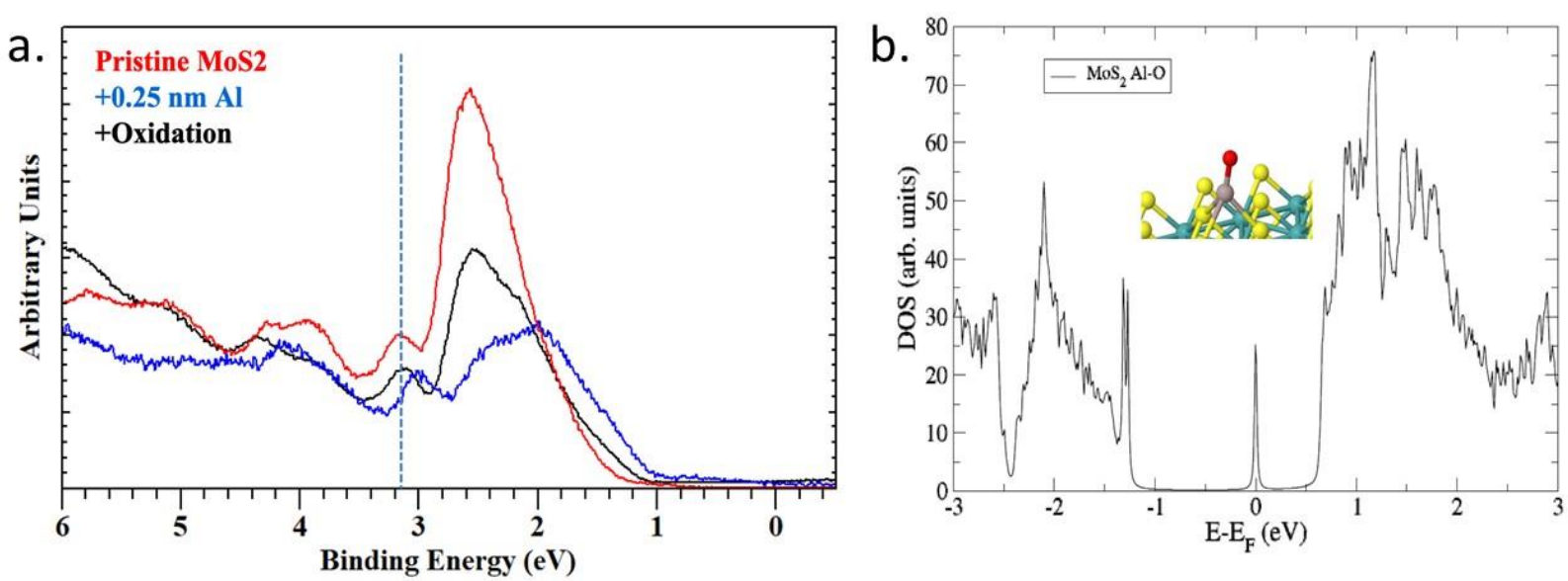

Figure S3. a. Valence band spectra acquired around gamma point (we have performed the summation of the spectra at constant momentum in function of E between -0.5 to $\left.0.5 \AA^{-1}\right)$. In red and blue before and after $0.25 \mathrm{~nm}$ Al deposition and in black after oxidation at 1 mbar for 1 h30 min. b. Density Functional Theory calculation of the bandstructure of the $\mathrm{MoS}_{2}-(\mathrm{S}-\mathrm{Al}-\mathrm{O})$ interface.

We have finally performed Density Functional Theory (DFT) calculations to elucidate the conformation and the bandstructure of the $\mathrm{Al}-\mathrm{MoS}_{2}$ interface, respect to these experimental results. To this end, we have used the very efficient localized orbital basis set DFT code Fireball ${ }^{[5]}$. Optimized basis sets for Mo, S, Al and $\mathrm{O}$ have been used, in agreement with previous calculations ${ }^{[6,7]}$. Hence, we have considered a $5 \times 5$ layer of $\mathrm{MoS}_{2}$, with an Al-O dimer on top, connected to a sulfur atom through either the aluminium atom or the Oxygen one. Both structures have been optimized, and as a result, the $\mathrm{S}-\mathrm{Al}$ interface appears to be more stable 
energetically than the S-O interface for about $2 \mathrm{eV}$. In the case of $\mathrm{MoS}_{2}-(\mathrm{S}-\mathrm{O}-\mathrm{Al})$, the band structure is strongly shifted toward Ef by $0.7 \mathrm{eV}$ while in the case of $\mathrm{MoS}_{2}-(\mathrm{S}-\mathrm{Al}-\mathrm{O})$ a P-doping is also observed with a lower energy shift $(0.1 \mathrm{ev})$. We observe also a feature around $1.3 \mathrm{eV}$ under Ef. Both the energy shift and the observed feature, strongly suggest that the interface is $\mathrm{MoS}_{2}-(\mathrm{S}-\mathrm{Al}-\mathrm{O})$. The DFT calculations reveal also that the feature around $1.3 \mathrm{eV}$ is associated to the presence of oxygen atom bonded to the $\mathrm{Al}$ at the interface (Figure $\mathbf{3 b}$ ).

We conclude from this combined analysis that the oxidation process of our ultrathin aluminium layer leads to the formation of core-shell nanostructures with a metallic core and an $\mathrm{Al}_{2} \mathrm{O}_{3}$ shell. In addition, the interface barrier between aluminium and $\mathrm{MoS}_{2}$ originates from the Al-S bonding, the oxidization process taking place at the surface of the deposited aluminium.

\section{Four points measurements of $\mathrm{MoS}_{2}$ channel}

We have performed four points measurements of the $\mathrm{MoS}_{2}$ channel. We observed metal-toinsulator transition. At lower $\mathrm{Vg}$, the four terminals conductance $\left(\mathrm{G}_{4 \mathrm{~T}}\right)$ increases with increasing T consistently with the typical semiconductor behaviour. At higher $\operatorname{Vg}(\geq 38)$ instead, $\mathrm{G}_{4 \mathrm{~T}}$ decreases with increasing $\mathrm{T}$ following a typical metallic behaviour. 

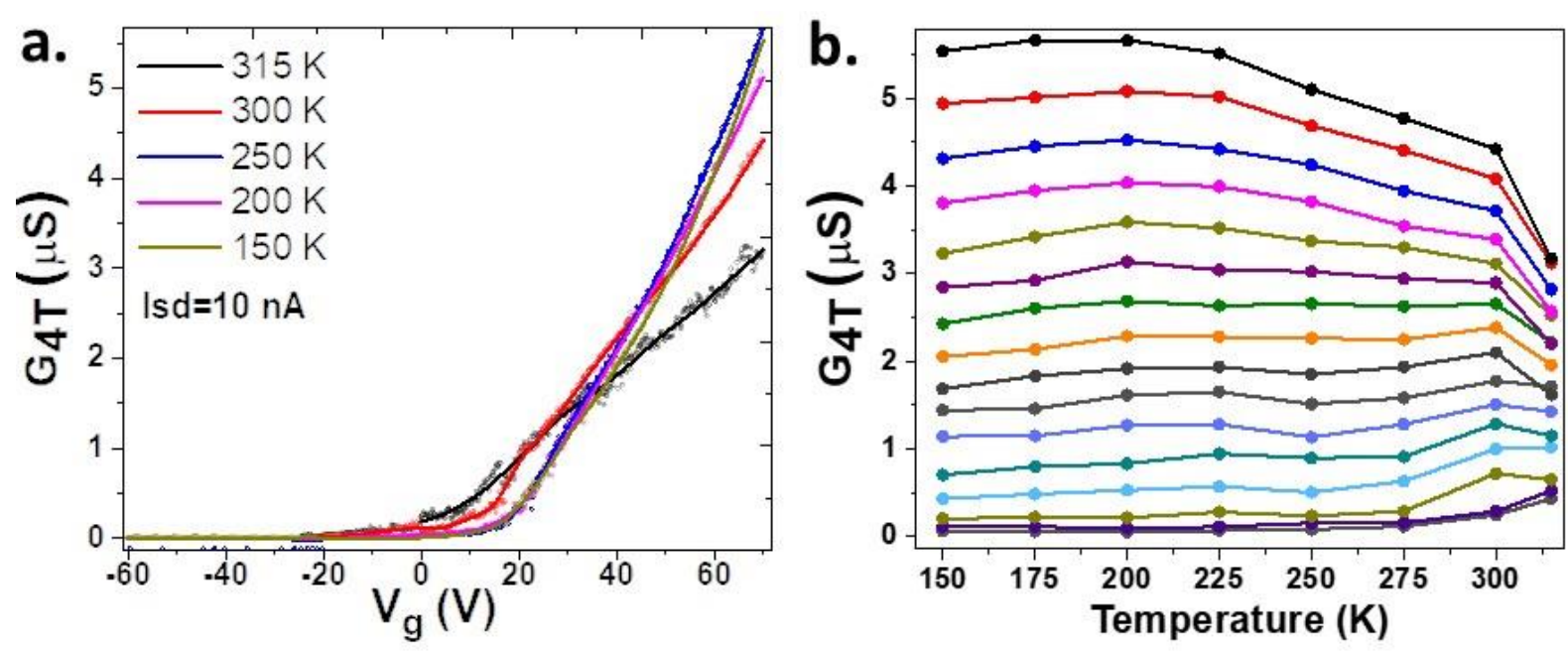

Figure S4. Metal-to-insulator transition in 20-OD Al-AlOx/MoS $\mathrm{S}_{2}$ based devices. a. Four terminals conductance $\left(G_{4 T}\right)$ vs. gate voltage $(V g)$ for an applied dc current of $10 \mathrm{nA}$. at different temperatures showing a metal to insulator crossing around Vg 38V b. Corresponding temperature dependency of $\mathrm{G}_{4 \mathrm{~T}}$ for $\mathrm{Vg}$ range of $14 \mathrm{~V}$ to $70 \mathrm{~V}$ from to bottom to top with and increment of $4 \mathrm{~V}$ for an applied dc current of $10 \mathrm{nA}$, demonstrating a gradual transition from an insulator to a metallic behaviour.

References :

[1] M. D. Siao, W. C. Shen, R. S. Chen, Z. W. Chang, M. C. Shih, Y. P. Chiu, C. M. Cheng, Nat. Commun. 2018, 9, 1.

[2] I. Horcas, R. Fernández, J. M. Gómez-Rodríguez, J. Colchero, J. Gómez-Herrero, A. M. Baro, Rev. Sci. Instrum. 2007, 78, 013705.

[3] G. Maidecchi, C. V. Duc, R. Buzio, A. Gerbi, G. Gemme, M. Canepa, F. Bisio, J. Phys. Chem. C 2015, 119, 26719.

[4] D. J. Trainer, A. V. Putilov, C. Di Giorgio, T. Saari, B. Wang, M. Wolak, R. U. Chandrasena, C. Lane, T. R. Chang, H. T. Jeng, H. Lin, F. Kronast, A. X. Gray, X. X. Xi, J. Nieminen, A. Bansil, M. Iavarone, Sci. Rep. 2017, 7, 1.

[5] J. P. Lewis, P. Jelínek, J. Ortega, A. A. Demkov, D. G. Trabada, B. Haycock, H. Wang, G. Adams, J. K. Tomfohr, E. Abad, H. Wang, D. A. Drabold, Phys. status solidi 2011, 248, 1989.

[6] M. A. Basanta, Y. J. Dappe, P. Jelínek, J. Ortega, Comput. Mater. Sci. 2007, 39, 759.

[7] S. Dubey, S. Lisi, G. Nayak, F. Herziger, V. D. Nguyen, T. Le Quang, V. Cherkez, C. González, Y. J. Dappe, K. Watanabe, T. Taniguchi, L. Magaud, P. Mallet, J. Y. Veuillen, R. Arenal, L. Marty, J. Renard, N. Bendiab, J. Coraux, V. Bouchiat, ACS Nano 2017, 11, 11206. 